\title{
Sparse Space-Time-Frequency-Domain Spreading for Large-Scale Non-Orthogonal Multiple Access
}

\author{
Yusha Liu, Student Member, IEEE, Lie-Liang Yang, Fellow, IEEE, and Lajos Hanzo, Fellow, IEEE
}

\begin{abstract}
A generalised spatial modulation-aided sparse space-time-frequency spreading (GSM/SSTFS) scheme is proposed for supporting large-scale access at a high normalized userload in next-generation systems by simultaneously exploiting the transmit diversity in the spatial- and frequency-domain. Specifically, in the GSM/SSTFS system employing two transmit antennas (TAs), each user equipment (UE) spreads its signal over two symbol durations, two active TAs, and multiple subcarriers by a unique pre-assigned sparse code, in order to attain spreading in three dimensions (3D). Furthermore, with the aid of GSM, extra information bits are embedded in the TA indices. At the receiver, a joint message passing-aided (JMPA) detector is employed for attaining near-single-user bit error rate (BER) performance, which is also compared to that of conventional multiple-input multiple-output non-orthogonal multiple access (MIMO-NOMA) systems.
\end{abstract}

Index Terms-Generalized Spatial Modulation, Space-Time Spreading, Message Passing.

\section{INTRODUCTION}

Next generation communication systems aim for supporting billions of devices in the Internet-of-Things (IoT) and in massive Machine-Type Communications (mMTC) [1,2]. However, facilitating massive access is challenging owing to the severe multi-user interference (MUI), especially for short packets. In this context, numerous power-domain or codedomain non-orthogonal multiple access (NOMA) schemes have been proposed for rank-deficient scenarios, where more user equipment (UEs) have to be supported than the number of resource-units [3-6]. While the power-domain NOMA exploits the different UE-powers and separates them by successive interference cancellation (SIC) at the receiver, code-domain NOMA schemes assign different non-orthogonal codes to UEs.

NOMA amalgamated with multiple-input multipleoutput (MIMO) techniques has been investigated in [7-11], including V-BLAST or generalised spatial modulation (GSM) [12]. At the time of writing, most studies of MIMONOMA rely on power-domain NOMA for downlink (DL) transmission. Since a pair of transmit antennas (TAs) has been standardized for the UE's uplink (UL) transmission

Copyright (c) 2015 IEEE. Personal use of this material is permitted. However, permission to use this material for any other purposes must be obtained from the IEEE by sending a request to pubs-permissions@ieee.org.

Y. Liu, L.-L. Yang and L. Hanzo are with School of Electronics and Computer Science, University of Southampton, SO17 1BJ, UK. (E-mail: yl6g15, 1ly, 1h@ecs.soton.ac.uk)

L. Hanzo would like to gratefully acknowledge the financial support of the Engineering and Physical Sciences Research Council projects EP/Noo4558/1, EP/PO34284/1, COALESCE, of the Royal Society's Global Challenges Research Fund Grant as well as of the European Research Council's Advanced Fellow Grant QuantCom. in the 3GPP Release 16 [13,14], substantial transmit diversity gain may be attained in UL multiuser MIMO transmission. A higher number of TAs may be employed in the context of massive MIMO techniques for next-generation communications [15]. However, there is a paucity of literature on simultaneously exploiting both the spatial- and frequencydomain diversity for enhancing the performance of NOMA systems. Motivated by achieving transmit diversity at the UE and inspired by the space-time spreading (STS) technique that was originally designed for code-division multiple-access (CDMA) systems [16], we propose a GSM-aided sparse space-time-frequency spreading (GSM/SSTFS) scheme for NOMA systems, in order to support large-scale access in next generation systems. The contributions of this paper are summarised as follows:

- For the first time, we propose a GSM/SSTFS scheme for supporting large-scale access at a high normalized user-load by involving STF-domain spreading. More specifically, in the GSM/SSTFS system relying on two active TAs, each UE spreads its signal over two symbol durations, two active TAs, and multiple subcarriers by a unique, user-specific sparse code, before transmitting the signal over the channel. Furthermore, with the aid of GSM, extra information bits are embedded in the TA indices, for increasing the attainable throughput.

- Secondly, a joint factor graph is designed for signal detection, which is eminently suitable for visualizing the message-propagation by the STF-domain spreading. Based on the connections of the proposed joint factor graph, a joint message passing-aided (JMPA) detector is conceived for attaining a near-single-user bit error rate (BER) at a low complexity.

- Finally, our BER performance results have demonstrated that the proposed GSM/SSTFS scheme achieves superior BER, compared to the conventional MIMO-NOMA schemes at the same number of bits per symbol (BPS).

The rest of this paper is structured as follows. Section II describes the transmitter and receiver of the proposed GSM/SSTFS system. Following this, Section III introduces our JMPA detection and the BER performance of the proposed GSM/SSTFS system is characterized in Section IV. Finally, our main conclusions and future research ideas will be discussed in Section V.

\section{GSM/SSTFS SYSTEM MOdEL}

In this section, the transmitter and receiver models of the proposed GSM/SSTFS UL are introduced in Sections II-A and 


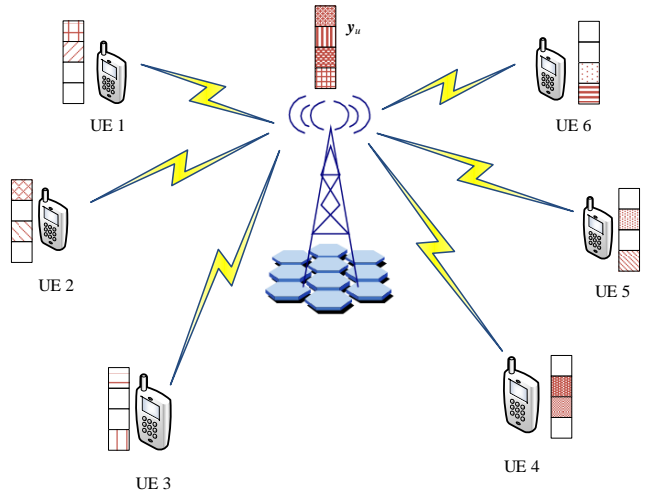

Fig. 1. An example of the uplink GSM/SSTFS network employing $N=4$ to support $K=6$ UEs.

II-B, respectively. In this paper, a single-cell UL MIMO multicarrier (MC) communication system is assumed, as shown in Fig. 1, where $K$ UEs simultaneously transmit their UL signals to a single BS by $N$ subcarriers $(K \geq N)$ over frequencyselective Rayleigh fading channels having $L$ resolvable paths.

\section{A. Transmitter Model}

At the transmitter, each of the $K$ UEs is equipped with $N_{T}$ TAs, whereas the BS at the receiver employs $U$ receive antennas (RAs). Furthermore, we assume that $N$ subcarriers $(N>L)$ are employed for transmitting the UL signal to the B$\mathrm{S}$, in line with the MC systems' typical design [17], so that the individual subcarriers experience flat fading, but the adjacent subcarriers may experience correlated fading. Following the principles of GSM, within each symbol duration, only $t$ TAs $\left(t<N_{T}\right)$ of a UE are activated. The GSM/SSTFS transmitter of UE $k$ is shown in Fig. 2.

More specifically, for UE $k$, a total of $b=b_{1}+b_{2}$ bits are transmitted in each symbol duration, where $b_{1}=\left\lfloor\log _{2}\left(\begin{array}{c}N_{T} \\ t\end{array}\right)\right\rfloor^{1}$ bits are mapped to the TA indices [12], where the GSM symbol set is expressed as $\mathcal{M}_{1}=\left\{p_{1}, \cdots, p_{M_{1}}\right\}$ and $M_{1}=2^{b_{1}}$. By contrast, the remaining $b_{2}=t \log _{2} M_{2}$ bits are conveyed by the $M_{2}$-QAM symbols of the set $\mathcal{M}_{2}=\left\{s_{1}, \cdots, s_{M_{2}}\right\}$. For instance, for a $\left(N_{T}=4, t=2\right)$ GSM system, we have $\left(\begin{array}{c}N_{T} \\ t\end{array}\right)=\left(\begin{array}{l}4 \\ 2\end{array}\right)=6$ TA combinations, which can be exploited for transmitting $b_{1}=2$ bits per GSM symbol, using the bit-symbol-TA mapping of Table I, where $t_{i} \in \mathcal{M}_{1}$ is the GSM symbol and $\left(n_{1}, n_{2}\right)$ are the indices of the active TAs. Furthermore, $b_{2}$ bits are mapped to $t M_{2}$-QAM symbols expressed as $\boldsymbol{s}_{k}=[\underbrace{s_{k, n_{1}}, s_{k, n_{2}}, \cdots, s_{k, n_{t}}}_{t}]^{T}$, where $s_{k, n_{i}} \in \mathcal{M}_{2}$, which are transmitted by the $n_{1^{-}}, \cdots, n_{t}$-th TAs, respectively.

As shown in Fig. 2, following the $M_{2}$-QAM mapping, SSTFS is applied to the $t M_{2}$-QAM symbols, in order to simultaneously achieve diversity in both the spatial-domain (SD), time-domain (TD) and frequency-domain (FD). In the following discussions, we only consider the case of $t=2$ active TAs, since employing a pair of RF chains has been

\footnotetext{
${ }^{1}\lfloor x\rfloor$ represents the largest integer that is smaller than $x$.
}

TABLE I

BIT-SYMBOL-TA MAPPING TABLE FOR THE GSM WITH $N_{T}=4, t=2$.

\begin{tabular}{c|c|c}
\hline $\boldsymbol{b}_{k 1}$ & $\mathcal{M}_{1}$ & $\left(n_{1}, n_{2}\right)$ \\
\hline \hline 00 & $p_{1}$ & $(1,3)$ \\
\hline 01 & $p_{2}$ & $(2,4)$ \\
\hline 10 & $p_{3}$ & $(1,4)$ \\
\hline 11 & $p_{4}$ & $(2,3)$ \\
\hline
\end{tabular}

adopted for MIMO UL transmission in the 3GPP Release 16 for 5G NR [13].

Firstly, sparse FD spreading is carried out by adopting the code-domain NOMA principles relying on low-density signatures (LDS) [18]. More specifically, a sparse spreading sequence $\boldsymbol{c}_{k}=\left[c_{k 1}, c_{k 2}, \ldots, c_{k N}\right]^{T}$ is preassigned to UE $k$ and it is normalized to satisfy $\left\|\boldsymbol{c}_{k}\right\|^{2}=1$. We assume that in $\boldsymbol{c}_{k}$, only $d_{x}\left(d_{x}<<N\right)$ of the $N$ elements are non-zero, which represent the number of subcarriers that UE $k$ spreads its signal over in the FD. For example, for a $N=4, K=6$ GSM/SSTFS system, the spreading matrix $\boldsymbol{F}$, which indicates the non-zero element-positions for all $K=6$ UEs can be expressed as

$$
\boldsymbol{F}=\left[\begin{array}{llllll}
1 & 1 & 1 & 0 & 0 & 0 \\
1 & 0 & 0 & 1 & 1 & 0 \\
0 & 1 & 0 & 1 & 0 & 1 \\
0 & 0 & 1 & 0 & 1 & 1
\end{array}\right]
$$

Correspondingly, the number of UEs sharing one of the $N$ subcarriers, i.e. the number of non-zero elements in each row of $\boldsymbol{F}$, is expressed as $d_{c}$, which has the property of $d_{c}<<K$.

In contrast to the conventional STS scheme [16], where two QAM symbols are spread across two TAs over two symbol durations by a dense spreading sequence, again, we adopt the sparse FD spreading sequence $\boldsymbol{c}_{k}$ for SD spreading. Specifically, for UE $k$, a pair of sparse spreading sequences are formed as

$$
\begin{aligned}
& \boldsymbol{c}_{k 1}=\frac{1}{\sqrt{2}}\left[\begin{array}{ll}
\boldsymbol{c}_{k}^{T} & \boldsymbol{c}_{k}^{T}
\end{array}\right]^{T}, \\
& \boldsymbol{c}_{k 2}=\frac{1}{\sqrt{2}}\left[\begin{array}{ll}
\boldsymbol{c}_{k}^{T} & -\boldsymbol{c}_{k}^{T}
\end{array}\right]^{T},
\end{aligned}
$$

Furthermore, the pair of symbols, $s_{k, n_{1}}$ and $s_{k, n_{2}}$, to be transmitted by the $n_{1}$-th and $n_{2}$-th activated TAs, respectively, are space-time coded (STC) by Alamouti's code $\mathcal{G}_{2}$ [19] as:

$$
\mathcal{G}_{k, 2}=\frac{1}{\sqrt{2}}\left[\begin{array}{cc}
s_{k, n_{1}} & s_{k, n_{2}} \\
-s_{k, n_{2}}^{*} & s_{k, n_{1}}^{*}
\end{array}\right] .
$$

Hence, after the STC and spreading, we have a $(2 N \times 2)$ dimensional transmit signal matrix $\boldsymbol{X}_{k}$ expressed as

$$
\begin{aligned}
\boldsymbol{X}_{k} & =\frac{1}{\sqrt{2}}\left[\begin{array}{ll}
\boldsymbol{c}_{k 1} & \boldsymbol{c}_{k 2}
\end{array}\right]\left[\begin{array}{cc}
s_{k, n_{1}} & s_{k, n_{2}} \\
-s_{k, n_{2}}^{*} & s_{k, n_{1}}^{*}
\end{array}\right] \\
& =\left[\begin{array}{ll}
\boldsymbol{x}_{k, n_{1}} & \boldsymbol{x}_{k, n_{2}}
\end{array}\right]
\end{aligned}
$$

where $\boldsymbol{x}_{k, n_{1}}$ and $\boldsymbol{x}_{k, n_{2}}$ are transmitted by the $n_{1}$-th and $n_{2}$-th TAs, respectively.

Additionally, the transmit signal of UE $k$ in a further generalized GSM/SSTFS system employing $t(t \geq 2)$ active TAs over $D$ symbol durations can be expressed as

$$
\begin{aligned}
\boldsymbol{X}_{k} & =\left[\begin{array}{llll}
\boldsymbol{c}_{k 1} & \boldsymbol{c}_{k 2} & \cdots & \boldsymbol{c}_{k D}
\end{array}\right] \mathcal{G}_{t}^{(k)} \\
& =\left[\begin{array}{llll}
\boldsymbol{x}_{k, n_{1}} & \boldsymbol{x}_{k, n_{2}} & \cdots & \boldsymbol{x}_{k, n_{t}}
\end{array}\right],
\end{aligned}
$$




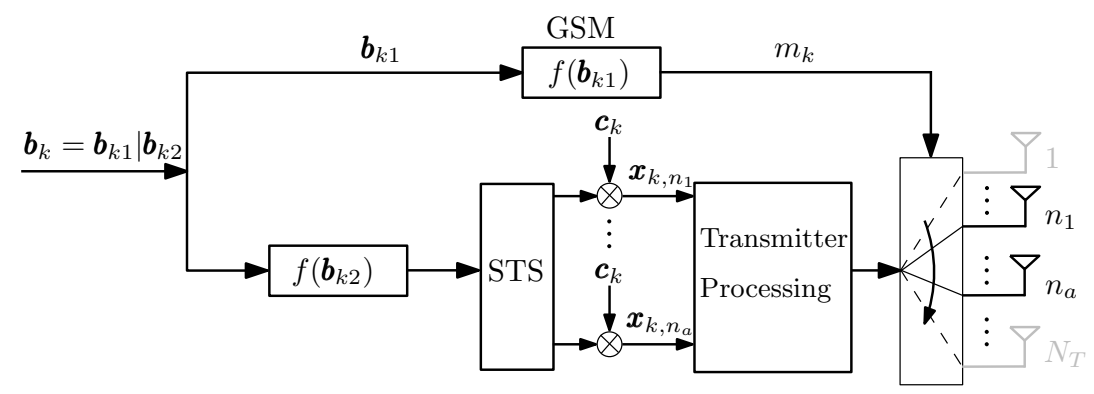

Fig. 2. The transmitter structure of the $k$ th UE in the GSM/SSTFS system.

where the design of $\left[\boldsymbol{c}_{k 1}, \cdots, \boldsymbol{c}_{k t}\right]$ is discussed in [17] and $\mathcal{G}_{t}^{(k)}$ represents a STC constructed by a block of symbols transmitted by the $k$-th UE, as shown in [16].

\section{B. Receiver Model}

The channel impulse response (CIR) between the $n_{i}$-th TA $\left(n_{i}=1,2, \cdots, N_{T}\right)$ of the $k$-th UE $(k=1,2, \cdots, K)$ and the $u$-th RA $(u=1,2, \cdots, U)$ of the BS is denoted by:

$$
\boldsymbol{h}_{k, n_{i}}^{(u)}=\left[h_{k, n_{i}, 1}^{(u)}, h_{k, n_{i}, 2}^{(u)}, \cdots, h_{k, n_{i}, L}^{(u)}\right]^{T},
$$

where $\boldsymbol{h}_{k, n_{i}}^{(u)}$ is independent identically distributed (iid) in terms of $k, n_{i}$, and $u$, and obeys the complex Gaussian distribution with zero mean and a variance of $0.5 / L$ per dimension. Then, the corresponding FD channel transfer function (FD-CHTF) experienced by the $N$ subcarriers can be expressed as [17]

$$
\hat{\boldsymbol{h}}_{k, n_{i}}^{(u)}=\mathcal{F} \boldsymbol{\Phi}_{L} \boldsymbol{h}_{k, n_{i}}^{(u)},
$$

where $\boldsymbol{\Phi}_{L}$ having a dimension of $(N \times L)$ is formed by the first $L$ columns of an identity matrix $\boldsymbol{I}_{N}$, while $\mathcal{F}$ represents the FFT having the property of $\mathcal{F F}^{H}=\mathcal{F}^{H} \mathcal{F}=N I_{N}$.

Following the assumptions routinely exploited in STS and STC [16], in the GSM-SSTFS system having $t=2$ we assume that the CIR $\boldsymbol{h}_{k, n_{i}}^{(u)}$ remains constant for two consecutive symbols.

Therefore, the observations received at the $u$-th RA over the 2 symbol durations of a GSM-SSTFS system can be expressed in (8), where $\boldsymbol{n}_{u}^{(i)}$ is additive white Gaussian noise (AWGN) with zero-mean and a covariance matrix of $2 \sigma^{2} \boldsymbol{I}_{N}$, expressed as $\mathcal{C} \mathcal{N}\left(0,2 \sigma^{2} \boldsymbol{I}_{N}\right)$, where $\sigma^{2}=1 /(2 \gamma), \gamma=b \gamma_{0}$ denotes the signal-to-noise ratio (SNR) per symbol, while $\gamma_{0}$ is the SNR per bit.

After assembling $\boldsymbol{y}_{u}^{(1)}$, which is the conjugate of $\boldsymbol{y}_{u}^{(2)}$ into a vector, the received $\left[\left(\boldsymbol{y}_{u}^{(1)}\right)^{T},\left(\boldsymbol{y}_{u}^{*(2)}\right)^{T}\right]^{T}$ can be expressed in (9), where $\boldsymbol{C}_{k}=\operatorname{diag}\left\{\boldsymbol{c}_{k}\right\}$ and $\boldsymbol{H}_{m_{k}}$ represents the channel matrix between the $t$ active TAs of UE $k$ selected by the GSM symbol $m_{k}$ and the receiver. Hence we have obtained the MIMO equation for our GSM-SSTFS system. Next, our JMPA detector is conceived in Section III-B.

\section{Joint Message Passing-Aided Detection}

Let us first express the symbols $\boldsymbol{s}_{k}=\left[s_{k, n_{1}}, \cdots, s_{k, n_{t}}\right]$, $s_{k, n_{t}} \in \mathcal{M}_{2}, n_{i}=n_{1}, \cdots, n_{t}$, transmitted by the $t$ active TAs of UE $k$ in the form of a combined constellation symbol set defined as $\mathcal{M}=\underbrace{\mathcal{M}_{2} \otimes \cdots \otimes \mathcal{M}_{2}}_{t}=\left\{g_{1}, g_{2}, \cdots, g_{M_{2}^{t}}\right\}$, which has a size of $M_{2}^{t}$. More explicitly, $g_{1}, g_{2}, \cdots, g_{M_{2}^{t}}$ represent the combinations of $t$ QAM symbols. In the case of $t=2$ and $\mathcal{M}_{2}=\{0,1\}$, we have $\mathcal{M}=\mathcal{M}_{2} \otimes \mathcal{M}_{2}=\{00,01,10,11\}$. Then the GSM-QAM symbols transmitted by the $k$-th UE employing $t$ active TAs can be expressed as $z_{k}=m_{k} \mid g_{k}$, where $m_{k} \in \mathcal{M}_{1}, g_{k} \in \mathcal{M}$ and $z_{k} \in \mathcal{M}_{1} \otimes \mathcal{M}$. Therefore, the GSM-QAM symbols transmitted by all $K$ UEs can be defined as $\boldsymbol{z}=\left[z_{1}, \ldots, z_{K}\right]$.

Let us now assume that the BS perfectly knows both the CIRs and the spreading sequences. Then, the maximumlikelihood detection (MLD) finds the estimate of $z$ by solving the optimization problem of

$$
\begin{aligned}
\hat{\boldsymbol{z}} & =\left[\hat{z}_{1}, \ldots, \hat{z}_{K}\right] \\
& =\left[\hat{m}_{1}\left|\hat{g}_{1}, \cdots, \hat{m}_{K}\right| \hat{g}_{K}\right] \\
& =\underset{\tilde{m}_{\tilde{1}}, \cdots, \tilde{m}_{K} \in \mathcal{M}_{1}}{\arg \min }\left\{\left\|\boldsymbol{y}-\sum_{k=1}^{K} \boldsymbol{H}_{\tilde{m}_{k}} \tilde{g}_{k}\right\|^{2}\right\},
\end{aligned}
$$

where the legitimate combinations of $\boldsymbol{H}_{\tilde{m}_{k}}$ and $\tilde{g}_{k}$ represent all possible candidates $\tilde{z}_{k}$. Explicitly, the MLD has to visit all possible candidate symbols in the symbol set $\mathcal{M} \otimes \mathcal{M}_{1}$ of all UEs, which has a detection complexity on the order of $\mathcal{O}\left[\left(M_{1} M_{2}^{t}\right)^{K}\right]$. This excessive-complexity MLD is unsuitable for practical GSM/SSTFS systems.

Therefore, a low-complexity JMPA detection algorithm is proposed in this section, which jointly detects the GSM-QAM symbol based on the message-passing principle. Hence, in Section III-A, we first design a factor graph for reducedcomplexity signal detection, followed by our JMPA detector conceived in Section III-B.

\section{A. Factor Graph Design}

Conventionally, a factor graph can be employed by a NOMA system for representing the connections between the UEs' transmitted symbols and the resource units transmitting these symbols. However, in a GSM/SSTFS system, a UE's signal is transmitted by both the classic QAM symbols and the TAs activated from the $N_{T}$ available TAs. Naturally, the receiver does not have the knowledge of the active TA indices before the detection. Hence, the corresponding factor graph has to be constructed to show the connections between the 


$$
\begin{gathered}
{\left[\begin{array}{c}
\boldsymbol{y}_{u}^{(1)} \\
\boldsymbol{y}_{u}^{(2)}
\end{array}\right]=\sum_{k=1}^{K}\left(\operatorname{diag}\left\{\boldsymbol{x}_{k, n_{1}}\right\}\left[\begin{array}{c}
\hat{\boldsymbol{h}}_{k, n_{1}}^{(u)} \\
\hat{\boldsymbol{h}}_{k, n_{1}}^{(u)}
\end{array}\right]+\operatorname{diag}\left\{\boldsymbol{x}_{k, n_{2}}\right\}\left[\begin{array}{c}
\hat{\boldsymbol{h}}_{k, n_{2}}^{(u)} \\
\hat{\boldsymbol{h}}_{k, n_{2}}^{(u)}
\end{array}\right]\right)+\left[\begin{array}{c}
\boldsymbol{n}_{u}^{(1)} \\
\boldsymbol{n}_{u}^{(2)}
\end{array}\right],} \\
\underbrace{\left[\begin{array}{c}
\boldsymbol{y}_{u}^{(1)} \\
\boldsymbol{y}_{u}^{*(2)}
\end{array}\right]}_{\boldsymbol{y}}=\sum_{k=1}^{K} \frac{1}{2} \underbrace{\left[\begin{array}{cc}
\boldsymbol{C}_{k} & \boldsymbol{C}_{k} \\
\boldsymbol{C}_{k} & -\boldsymbol{C}_{k}
\end{array}\right]\left[\begin{array}{cc}
\hat{\boldsymbol{h}}_{k, n_{1}}^{(u)} & \hat{\boldsymbol{h}}_{k, n_{2}}^{(u)} \\
\hat{\boldsymbol{h}}_{k, n_{2}}^{*(u)} & -\hat{\boldsymbol{h}}_{k, n_{1}}^{*(u)}
\end{array}\right]}_{\boldsymbol{H}_{m_{k}}} \underbrace{\left[\begin{array}{c}
s_{k, n_{1}} \\
s_{k, n_{2}}
\end{array}\right]}_{\boldsymbol{s}_{k}}+\underbrace{\left[\begin{array}{c}
\boldsymbol{n}_{u}^{(1)} \\
\boldsymbol{n}_{u}^{*(2)}
\end{array}\right]}_{\boldsymbol{n}} .
\end{gathered}
$$

$K$ UEs' integrated symbols in the form of $m_{k} \mid g_{k}$ and the $N$ subcarriers, so that joint STF-domain detection can be performed.

As an example, the factor graph of a GSM/SSTFS system having $N=4, t=2, N_{T}=4, d_{x}=2$ and $d_{c}=3$, supporting $K=6 \mathrm{UEs}$ is illustrated in Fig. 3. Explicitly, the variable node (VN) $k^{(l)}$ represents the combined QAM symbol $g_{k}$ transmitted by the legitimate TA combination associated with the spatial symbol $m_{k} \in \mathcal{M}_{1}$ of UE $k$ in the $l$-th symbol duration, which is determined by the activated TAs of UE $k$. By contrast, the check node $(\mathrm{CN}) j(1 \leq j \leq N)$ represents the $j$-th subcarrier transmitting the signals of $d_{c}$ UEs.

Let us define the connections with the VNs and those with the CNs as

$$
\begin{aligned}
& \mathcal{V}_{k}=\left\{j: 1 \leqslant j \leqslant N, e_{k, j} \neq 0\right\}, k=1, \cdots, K \\
& \mathcal{C}_{j}=\left\{k: 1 \leqslant k \leqslant K, e_{k, j} \neq 0,\right\}, j=1, \cdots, N,
\end{aligned}
$$

where $e_{k, j}$ represents the connection between the $\mathrm{VN} k$ and the $\mathrm{CN} j$. To elaborate a little further, $\mathcal{V}_{k}$ collects all the CNs connected to the VN $k$ and $\mathcal{C}_{j}$ contains all the VNs connected to the $\mathrm{CN} j$.

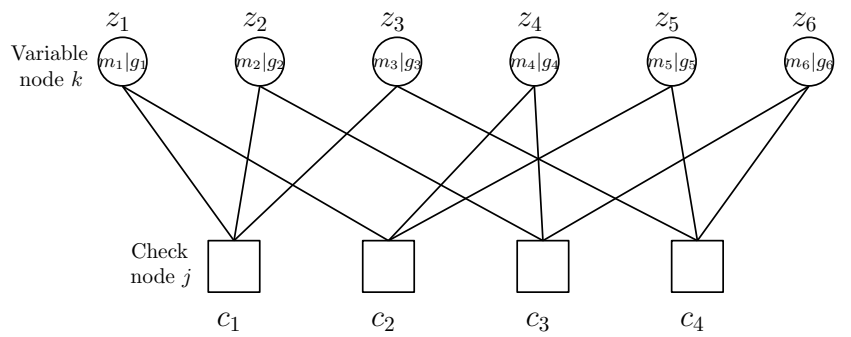

Fig. 3. An example of the factor graph representation of the GSM/SSTFS system using regular sparse sequences and the parameters of $N=4, n_{a}=2$, $d_{x}=2, d_{c}=3$ and $K=6$.

\section{B. Joint Message Passing-Aided Detection}

As shown in Fig. 3, information can be conveyed via the edge $e_{j, k}$ upward from the $\mathrm{CN} j$ to the $\mathrm{VN} k$. By contrast, information is conveyed from the $\mathrm{VN} k$ to the $\mathrm{CN} j$. The probability $\delta_{j, k}^{q, i}, q \in \mathcal{M}_{1} \otimes \mathcal{M}$ is the information conveyed from the $\mathrm{CN} j$ to the $\mathrm{VN} k$ during the $i$-th iteration. More explicitly, $\delta_{j, k}^{q, i}$ represents the probability of $z_{k}=q, q \in \mathcal{M}_{1} \otimes \mathcal{M}$, given the probabilities received by the $\mathrm{CN} j$ from all the connected VNs, but excluding $k$. Similarly, the information forwarded from the $\mathrm{VN} k$ to the $\mathrm{CN} j$ in the $i$-th iteration is denoted by $\eta_{k, j}^{q, i}$, which is defined as the probability of $z_{k}=q$, given the probabilities received by the VN $k$ from all the connected CNs, but excluding $j$. Then the JMPA detector performs the following steps.

First, $\eta_{k, j}^{q, 0}$ is initialised to $1 /\left(M_{1} M_{2}^{a}\right)$ for all $q \in \mathcal{M}_{1} \otimes \mathcal{M}$, and any $e_{k, j} \neq 0$. Then, at the $i$-th iteration, $\delta_{j, k}^{q, i}$ for $k \in \mathcal{V}_{k}$, and $j \in \mathcal{C}_{j}$ can be updated as

$$
\begin{aligned}
\delta_{j, k}^{q, i}= & \sum_{\boldsymbol{z}_{[j]} \in\left(\mathcal{M}_{1} \otimes \mathcal{M}\right)^{d_{c}-1}, z_{k}=q}\left(\prod_{z_{v} \in \boldsymbol{z}_{[j]} \backslash z_{k}} \eta_{k, j}^{z_{v}, i}\right) \\
& \times \prod_{u=1}^{U} \prod_{t=1}^{T} p\left(y_{u j}^{(l)} \mid z_{[j]}, z_{k}=q\right),
\end{aligned}
$$

where $\prod_{z_{v} \in \boldsymbol{z}_{[j]} \backslash z_{k}} \eta_{k, j}^{z_{v}, i}$ is the a priori probability of a given $z_{[j]}$ with $z_{k}=q$. For the GSM/SSTFS having $t=2$ TAs, when $\boldsymbol{z}_{[j]}$ is given, the PDF of $P\left(y_{u j}^{(l)} \mid \boldsymbol{z}_{[j]}\right)$ at the $l=1$-st and $l=2$-nd symbol duration can be expressed in (13) and (14), respectively.

Observe from (12) that the information conveyed by the $\mathrm{CN} j$ to the $\mathrm{VN} k$ is the product of the information gleaned from all the other edges connected to the $\mathrm{CN} j$. The total information conveyed to the $\mathrm{VN} k$ is the sum of the information arriving from all the $\mathrm{CNs}$ connected to the $\mathrm{VN} k$.

Next, at the $(i+1)$-st iteration, the values $\delta_{j, k}^{q, i}$ obtained in the $i$-th iteration are used for updating $\eta_{k, j}^{q, i+1}$ for $k \in \mathcal{V}_{k}$ and $j \in \mathcal{C}_{j}$ as follows

$$
\eta_{k, j}^{q, i+1}=\varphi_{k, j} \prod_{v \in \mathcal{V}_{k} \backslash k} \delta_{j, v}^{q, i},
$$

where $\varphi_{k, j}$ is the normalisation factor ensuring that $\sum_{\in \mathcal{M}_{1} \otimes \mathcal{M}} \eta_{k, j}^{q, i+1}=1$

Finally, after $I$ iterations, the symbol transmitted by the $k$-th UE can be expressed as

$$
\hat{z}_{k}=\hat{m}_{k} \mid \hat{g}_{k}=\underset{q \in \mathcal{M}_{1} \otimes \mathcal{M}}{\arg \max } \prod_{v \in \mathcal{V}_{k}} \delta_{v, k}^{q, i}, k=1,2, \ldots, K .
$$

Observe from (12) that the computational complexity is primarily determined by the upward information transition of Fig. 3 and, in particular, by the number of multiplications in (12). Therefore, the size of $z_{[j]}$ is employed to represent the complexity order of the JMPA detector, which comprises $\left(M_{1} M_{2}^{t}\right)^{d_{c}-1}$ legitimate combinations in $\left(\mathcal{M}_{1} \otimes \mathcal{M}\right)^{d_{c}-1}$. Hence, the complexity order of the JMPA detector can be expressed as $\mathcal{O}\left[\left(M_{1} M_{2}^{t}\right)^{d_{c}-1}\right]$. 


$$
\begin{aligned}
& P\left(y_{u j}^{(1)} \mid \boldsymbol{z}_{[j]}\right)=\frac{1}{2 \pi \sigma^{2}} \times \exp \left(-\frac{\left\|y_{u j}^{(1)}-\frac{1}{2} \sum_{k \in \mathcal{C}_{j}}\left\{c_{k j}\left[\hat{h}_{k, n_{1}, j}^{(u)}\left(s_{k, n_{1}}-s_{k, n_{2}}^{*}\right)+\hat{h}_{k, n_{2}, j}^{(u)}\left(s_{k, n_{2}}+s_{k, n_{1}}^{*}\right)\right]\right\}\right\|^{2}}{2 \sigma^{2}}\right), \\
& P\left(y_{u j}^{(2)} \mid \boldsymbol{z}_{[j]}\right)=\frac{1}{2 \pi \sigma^{2}} \times \exp \left(-\frac{\left\|y_{u j}^{(2)}-\frac{1}{2} \sum_{k \in \mathcal{C}_{j}}\left\{c_{k j}\left[\hat{h}_{k, n_{1}, j}^{(u)}\left(s_{k, n_{1}}+s_{k, n_{2}}^{*}\right)+\hat{h}_{k, n_{2}, j}^{(u)}\left(s_{k, n_{2}}-s_{k, n_{1}}^{*}\right)\right]\right\}\right\|^{2}}{2 \sigma^{2}}\right) .
\end{aligned}
$$

\section{Performance Results}

In this section, we quantify the BER performance of the proposed GSM/SSTFS scheme for transmission over frequencyselective Rayleigh fading channels having different number of CIR taps. Then we will compare the proposed GSM/SSTFS scheme both to the SSTFS scheme operating without GSM and to the MIMO-SCMA systems of [20] integrated with GSM. The number of active TAs is fixed to $t=2$, as in the $5 \mathrm{G}$ $\mathrm{NR}$, and each UE spreads the signal over $d_{x}=2$ subcarriers. Furthermore, the number of JMPA detection iterations is fixed to $I=15$, since usually the BER of the proposed GSM/SSTFS system no longer improves beyond $I=10$ iterations.

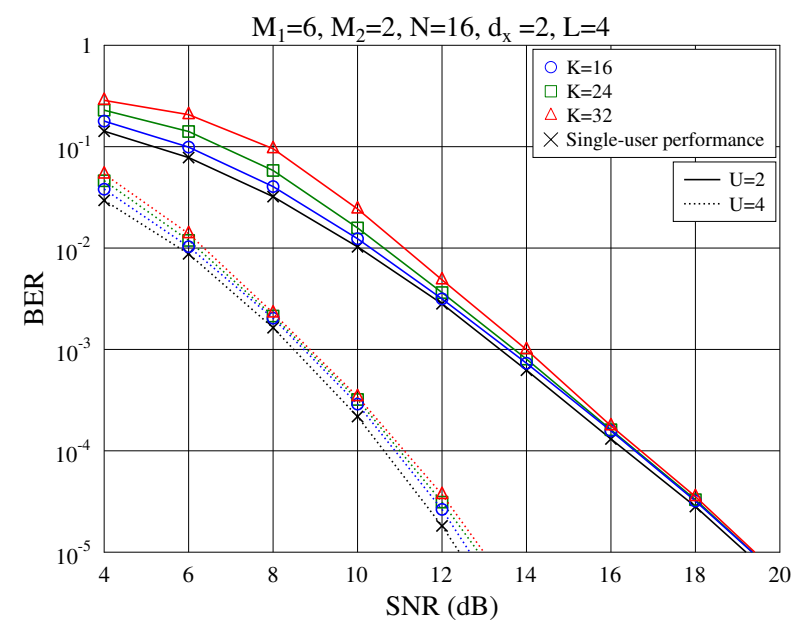

Fig. 4. BER performance of the GSM/SSTFS systems supporting $K=$ 16, 24 and 32 UEs within $N=16$ resource slots employing $U=2,4$ RAs over frequency-selective fading channels having $L=4$ CIR taps.

Firstly, the BER performance of the GSM/SSTFS system supporting the normalized UE loads of up to $\frac{K}{N}=200 \%$ is shown in Fig. 4 for transmission over frequency-selective fading channels having $L=4$ paths, $N=16$ subcarriers, $N_{T}=6$ TAs and binary phase shift keying (BPSK) modulation. The BER of the GSM/SSTFS system supporting a single user is also included as a benchmark. At the receiver, $U=2$ or $U=4$ receive antennas (RAs) are employed, which demonstrate a beneficial diversity gain at the receiver. Observe from Fig. 4 that the GSM/SSTFS system having a 200\% UE load shows a slight performance loss of about $1 \mathrm{~dB}$ compared to that of the $100 \%$ UE load at a BER level of $10^{-2}$. However, this BER performance loss becomes insignificant in higher signalto-noise ratios (SNR), where the BER of the GSM/SSTFS systems supporting $K=16,24$ and 32 UEs converge to that of the single-user scenario. Therefore, it can be concluded that with the aid of transmit diversity, the proposed GSM/SSTFS system becomes capable of supporting up to $200 \%$ UE load at a near-single-user BER in heavily-loaded next-generation systems.

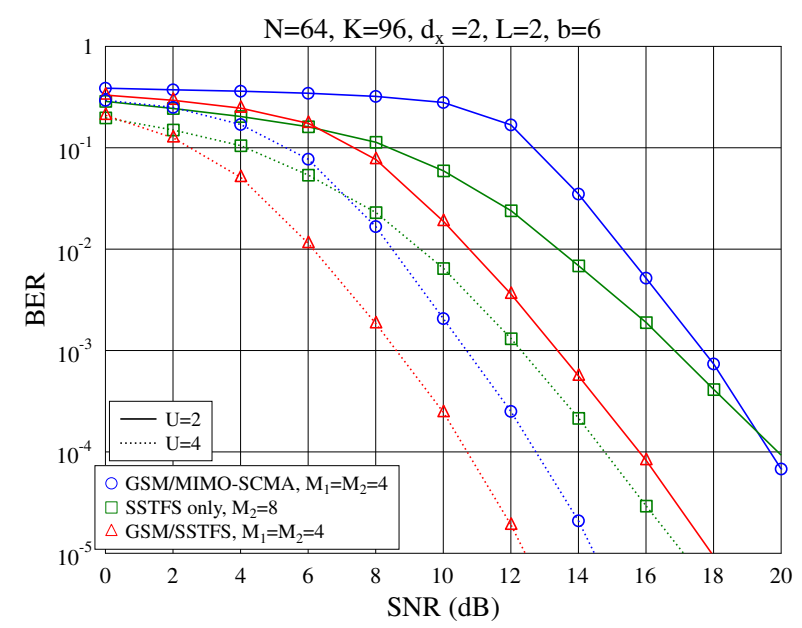

Fig. 5. BER comparison of the GSM/SSTFS, SSTFS, and GSM/MIMOSCMA systems with $U=2,4$ RAs supporting $K=96$ UEs within $N=64$ resource slots over frequency-selective fading channels having $L=2 \mathrm{CIR}$ taps.

Furthermore, we compare the BER performance of the GSM/SSTFS system to that of 1) SSTFS operating without GSM, and 2) the MIMO-SCMA system of [20] amalgamated with GSM at an identical data rate of $b=6 \mathrm{bps}$, when communicating over frequency-selective Rayleigh fading channels having $L=2$ or $L=16$ channel impulse response (CIR) taps, as shown in Figs. 5 and 6, respectively. In both figures, a total of $N=64$ subcarriers are employed for supporting $K=96$ UEs, giving a normalized UE load of $\frac{K}{N}=150 \%$. It can be observed from both Figs. 5 and 6 that the proposed GSM/SSTFS system achieves the best BER among the three candidate schemes considered. More specifically, for the SSTFS system dispensing with GSM, the 6-bit information is transmitted by only a pair of $M_{2}=8$-QAM symbols, whereas the proposed GSM/SSTFS scheme exploits the extra resource of the spatial domain, which maps the 6-bit information bits into a single 2-bit GSM symbol and a pair of $M_{2}=4$-QAM symbols. This achieves $4 \mathrm{~dB}$ gain at a BER level of $10^{-4}$, when communicating over frequency-selective fading channels having $L=16 \mathrm{CIR}$ taps and $U=2 \mathrm{RAs}$ are employed at the 


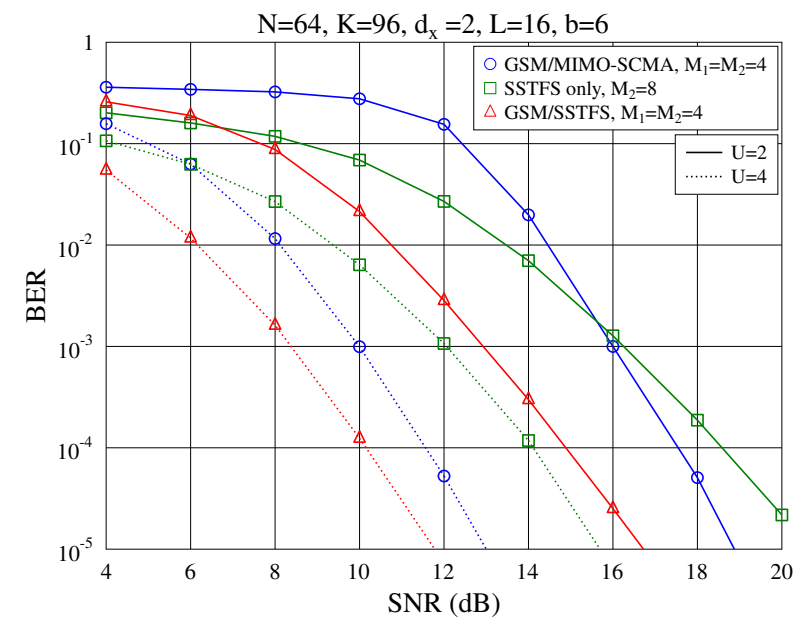

Fig. 6. BER comparison of the GSM/SSTFS, SSTFS, and GSM/MIMOSCMA systems with $U=2,4$ RAs supporting $K=96$ UEs within $N=64$ resource slots over frequency-selective fading channels having $L=16 \mathrm{CIR}$ taps.

receiver, as shown in Fig. 5.

By contrast, as shown in Fig. 6, in the case of a $150 \%$ UE load and $U=2$ RAs, the GSM/SSTFS system achieves up to $2.5 \mathrm{~dB}$ SNR gain at a BER of $10^{-4}$ over the GSMaided MIMO-SCMA system of [20] in the frequency-selective Rayleigh fading channels having $L=16$ CIR taps, which is achieved by the beneficial exploitation of STF-domain spreading. Hence, the GSM/SSTFS scheme may find promising application in large-scale access scenarios, where a large number of UEs access a single access point (AP) via limited spectral resources. Additionally, by comparing Figs. 5 and 6, we can see the advantageous frequency diversity gain of MC communications for transmission over frequency-selective channels, where all the systems achieve superior BER performance.

\section{Conclusions}

We have proposed a GSM/SSTFS scheme relying on the principle of NOMA for supporting large-scale access in nextgeneration wireless systems by simultaneously exploiting the transmit diversity gleaned from the SD and FD. Specifically, in the GSM/SSTFS system employing two active TAs, each UE spreads its signal over two symbol durations, two active TAs and multiple subcarriers. Extra information bits are embedded in the TA indices with the aid of GSM. Hence, the GSM/SSTFS scheme allows each UE to transmit at a relatively high data rate, in addition to supporting large-scale access at a high normalized UE-load. Furthermore, our JMPA detector imposes a low complexity. Our simulation results show that compared to the conventional MIMO-NOMA scheme, the proposed GSM/SSTFS system is capable of achieving a 5$\mathrm{dB}$ SNR gain at $6 \mathrm{bps}$ and at a BER of $10^{-5}$, in the case of $N=64$ and $K=96$, i.e. at a normalized loading factor of $150 \%$.

An interesting future research topic may be grant-free transmission based on our GSM/SSTFS system relying on joint channel estimation, UE activity and data detection. Additionally, further potential research issues include the design and optimization of the spreading sequences of GSM/SSTFS systems.

\section{REFERENCES}

[1] L. Liu and W. Yu, "Massive connectivity with massive MIMO-Part I: Device activity detection and channel estimation," IEEE Transactions on Signal Processing, vol. 66, no. 11, pp. 2933-2946, 2018.

[2] L. Tello-Oquendo, V. Pla, I. Leyva-Mayorga, J. Martinez-Bauset, V. Casares-Giner, and L. Guijarro, "Efficient random access channel evaluation and load estimation in LTE-A with massive MTC," IEEE Transactions on Vehicular Technology, vol. 68, no. 2, pp. 1998-2002, 2018.

[3] Y. Liu, Z. Qin, M. Elkashlan, Z. Ding, A. Nallanathan, and L. Hanzo, "Nonorthogonal multiple access for $5 \mathrm{G}$ and beyond," Proceedings of the IEEE, vol. 105, no. 12, pp. 2347-2381, Dec 2017.

[4] M. Moltafet, N. M. Yamchi, M. R. Javan, and P. Azmi, "Comparison study between PD-NOMA and SCMA," IEEE Transactions on Vehicular Technology, vol. 67, no. 2, pp. 1830-1834, 2017.

[5] X. Li, J. Li, Y. Liu, Z. Ding, and A. Nallanathan, "Residual transceiver hardware impairments on cooperative noma networks," IEEE Transactions on Wireless Communications, 2019.

[6] H. Liu, T. A. Tsiftsis, K. J. Kim, K. S. Kwak, and H. V. Poor, "Rate splitting for uplink noma with enhanced fairness and outage performance," IEEE Transactions on Wireless Communications, 2020.

[7] Z. Ding, R. Schober, and H. V. Poor, "A general MIMO framework for NOMA downlink and uplink transmission based on signal alignment," IEEE Transactions on Wireless Communications, vol. 15, no. 6, pp. 4438-4454, 2016.

[8] X. Wang, J. Wang, L. He, and J. Song, "Spectral efficiency analysis for downlink NOMA aided spatial modulation with finite alphabet inputs," IEEE Transactions on Vehicular Technology, vol. 66, no. 11, pp. 10562 $10566,2017$.

[9] P. Yang, Y. Xiao, M. Xiao, and Z. Ma, "NOMA-aided precoded spatial modulation for downlink MIMO transmissions," IEEE Journal of Selected Topics in Signal Processing, vol. 13, no. 3, pp. 729-738, 2019.

[10] Y. Yu, H. Chen, Y. Li, Z. Ding, L. Song, and B. Vucetic, "Antenna selection for MIMO nonorthogonal multiple access systems," IEEE Transactions on Vehicular Technology, vol. 67, no. 4, pp. 3158-3171, 2017.

[11] Y. Liu, L. Yang, P. Xiao, H. Haas, and L. Hanzo, "Spatial modulated multicarrier sparse code-division multiple access," IEEE Transactions on Wireless Communications, vol. 19, no. 1, pp. 610-623, Jan 2020.

[12] J. Wang, S. Jia, and J. Song, "Generalised spatial modulation system with multiple active transmit antennas and low complexity detection scheme," IEEE Transactions on Wireless Communications, vol. 11, no. 4, pp. 1605-1615, 2012.

[13] 3GPP Technical Report 21.916, "Release 16 description; summary of Rel-16 work items," 3rd Generation Partnership Project, 2019.

[14] H. Holma, A. Toskala, and T. Nakamura, 5G Technology: 3GPP New Radio. John Wiley \& Sons, 2020.

[15] M. Khani, M. Alizadeh, J. Hoydis, and P. Fleming, "Adaptive neural signal detection for massive mimo," IEEE Transactions on Wireless Communications, 2020.

[16] L.-L. Yang and L. Hanzo, "Performance of broadband multicarrier DSCDMA using space-time spreading-assisted transmit diversity," IEEE Transactions on Wireless Communications, vol. 4, no. 3, pp. 885-894, 2005.

[17] L.-L. Yang, Multicarrier Communications. Chichester, United Kingdom: John Wiley, 2009.

[18] R. Hoshyar, F. P. Wathan, and R. Tafazolli, "Novel low-density signature for synchronous CDMA systems over AWGN channel," IEEE Transactions on Signal Processing, vol. 56, no. 4, pp. 1616-1626, 2008.

[19] S. M. Alamouti, "A simple transmit diversity technique for wireless communications," IEEE Journal on Selected Areas in Communications, vol. 16, no. 8, pp. 1451-1458, 1998.

[20] J. Dai, K. Niu, and J. Lin, "Iterative Gaussian-approximated message passing receiver for MIMO-SCMA system," IEEE Journal of Selected Topics in Signal Processing, vol. 13, no. 3, pp. 753-765, 2019. 